\section{Microorganism transport from Earth to Mars}

SIR-In a recent letter, Melosh' presented the concept that large impacts on the Earth may eject rocks containing microorganisms that could fall on Mars or other planets. I have independently developed a similar model, and in November 1987 I submitted a very long article, which Nature was unable to publish. Here I would like to compare the two models. I will use the term dust/solar pressure/keplerian model (DSPK) to refer to my work and boulder/keplerian model (BK) when referring to Melosh's work; the meanings of these terms will become evident in what follows.

The DSPK model focuses on the transport of microorganisms from the Earth to Mars. This model starts with a meteorite impact on the Earth that can launch into space debris containing microorganisms from the surface of the planet. A fraction of this ejecta goes into orbit around the planet and some small fraction reaches

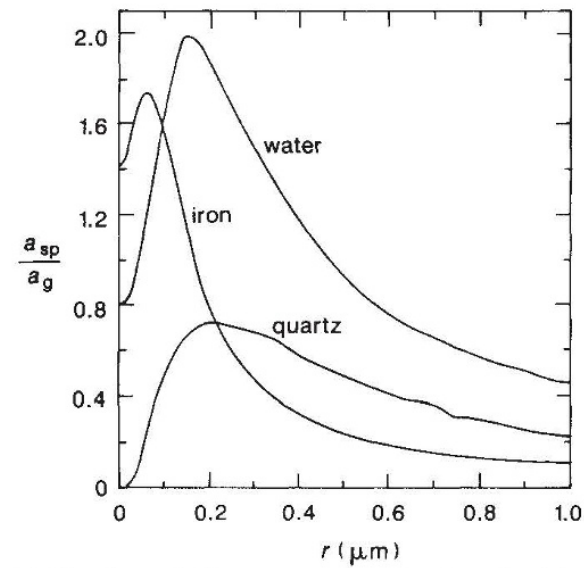

Ratio of sunlight-pressure acceleration $\left(a_{\mathrm{sp}}\right)$ to solar gravitational attraction $\left(a_{\mathrm{p}}\right)$ as a function of particle radius $(r)$ for various materials ${ }^{+}$.

escape velocity. The small particles $(<0.5$ $\mu \mathrm{m})$ in the fraction that escapes the gravitational influence of the planet and is placed in Earth's orbit beyond $45 R_{+}$ (where $R_{\oplus}$ is the Earth radius) can reach Mars by means of solar light pressure as a propulsion mechanism²; larger particles may reach Mars by means of keplerian trajectories. The converse of the latter can be seen in the case of the SNC meteorites, which are thought to have come from Mars.

The timescale for the transport of microscopic particles from Earth to Mars by the solar light pressure mechanism is about two months, whereas the bouldersized rocks proposed by Melosh as the carriers of microorganisms take, on average, a few million years to reach Mars. Laboratory and other evidence do not support the idea that microorganisms could last in viable form for a million years in such a hostile environment. Furthermore we can expect that radiation from radioactive elements present in the rock itself would destroy the microorganisms over such long periods of time. The mechanism of propulsion by solar radiation port mechanism, but places the constraint that only particles with a diameter of 0.5 $\mu \mathrm{m}$ or less will be transported ${ }^{4}$ (see figure). Since there are many microorganisms with diameters of $\sim 0.01 \mu \mathrm{m}$, each dust particle could transport up to 50 such entities.

Another important feature considered in the DSPK model is that particles with diameters between $0.5 \mu \mathrm{m}$ and $1 \mathrm{~cm}$ that have reached escape velocity are many orders of magnitude more abundant than boulder-sized rocks and are distributed over a dust cloud several thousand kilometres in diameter when leaving the Earth. The probability of impact of such a cloud on Mars is up to $\sim 10^{16}$ times greater than for the boulders of $\sim 1 \mathrm{~m}$ in diameter proposed by Melosh. Another important difference between the two models is that the DSPK model includes a consideration of the environment met by the transported microorganisms. The presence of liquid water on Mars in the past ${ }^{5}$ suggests that at least at some time the transported microorganisms may have met with an environment favourable to their survival. Future Mars missions may thus find either fossils or live microorganisms and other forms of life in the north polar region, where there are still substantial amounts of water ice.

Uriburu 26 Sur,

Miguel. A. Moreno

Pocito 5427, San Juan,

Argentina

\section{Mclosh H.J Nature 332, 687-688 (1988).}

Bclton. M.J S Science 151, 35-44 (1966)

Mitchell, F.J. \& Ellis. W.L. Analysis of Surveyor 3 Material and Photographs Returned by Apollo 12 239-248 (1972).

4. Shapiro. I.I.. Lautman, D.A. \& Colombo. G. J. geophys. Res. 71, 5695-5704 (1966)

Carr. M. Icarus 68, 187--216(1986)

\section{Did God make RNA?}

SIR-The recent dispute between Maizels and Weiner' and Benner and Ellington' on the properties of primeval 'ribo-organisms' illustrates the extent to which the picture of a protein-less 'RNA world' has captured the imagination of molecular biologists. What was only a cautiously advanced hypothesis some years ago has now gained so much credibility as to go largely unquestioned. It is even presented to students as "almost certain". Some measure of balance needs to be restored, at least for the benefit of those who are not experts in the field.

If RNA came first, how did it arise? This crucial question is rarely raised. When it is, the astonishing admission is made that there is no plausible answer. Even committed proponents of the RNApressure is much more efficient as a trans- first creed acknowledge that "the accumulation of substantial quantities of relatively pure mononucleotides on the primitive Earth is highly implausible". As a way out, the authors postulate the prior formation of a somewhat less demanding oligonucleotide analogue which, however, still poses a formidable challenge to prebiotic ingenuity. Until the RNA world is provided with more solid foundations, its present vogue hardly seems justified. At the very least, appropriate consideration deserves to be given to alternative theories, of which there are several. A brief outline of some proposals that I formulated recently ${ }^{5-8}$ is given below.

My key argument is that nothing short of an extensive collection of catalysts, similar, be it in very crude form, to presentday enzymes, could have accounted for the prebiotic synthesis of such complex molecules as nucleotides. Given this premise, my model requires as sole starting materials the kind of simple amino acids and other small organic molecules that are readily obtained in abiotic simulation experiments or found in meteorites. Further chemical development is all accomplished through catalysis, leading to the progressive emergence of a network of protometabolic pathways, which I visualize as not very different from existing metabolism. Thus, the model, unlike most others, makes minimal demands on undirected abiotic chemistry.

I see the catalysts that are the main props of the model as rare members of a large population of randomly assembled oligopeptides and other multimers, from which active molecules could have emerged selectively thanks to substrate stabilization ${ }^{5}$. I view them as the primitive protoenzymes that must, for statistical reasons, be postulated as the precursors of present-day enzymes (see, for example, ref. 9). They could be ancestral to 'miniproteins' now encoded by exons (see, for example, ref. 10). I imagine their assembly as having proceeded from thioesters, which energetic considerations indicate could have been present in small, but significant, amounts in the kind of sulphur-rich, hot and acidic medium that many authors identify as the probable cradle of life (see, for example, ref. 11). Such thioesters could also have fuelled all the endergonic steps of protometabolism.

The possibility that a 'thioester world' may have preceded the RNA world is consistent with biochemical knowledge, as well as with the views of many biochemists. Indeed, the thioester bond lies at the very heart of life. It is the precursor of the pyrophosphate bond in key substratelevel phosphorylation processes, of virtually all naturally occurring ester bonds, and of numerous carbon-carbon bonds, such as citrate, fatty acids porphyrins, terpenes and steroids. It is particularly 